\title{
Value of Programmed Ventricular Stimulation in Presumed Carotid Sinus Syndrome
}

\author{
STEVEN D. NELSON, MD, WILLIAM H. KOU, MD, MICHAEL DE BUITLEIR, MD, \\ LORENZO A. DICARLO, Jr., MD, and FRED MORADY, MD
}

This study determines the results of programmed stimulation in patients with syncope or near-syncope presumed to have the carotid sinus śyndrome based on the finding of carotid sinus hypersensitivity and the absence of any other apparent cause for syncope or near-syncope after clinical evaluation. Fourteen patients had coronary artery disease, 1 had dilated cardiomyopathy and 18 patients did not have structural heart disease. Programmed simulation was performed at 2 basic drive cycle lengths and 2 right ventricular sites with 1 to 3 extrastimuli. Sustained unimorphic ventricular tachycardia (VT) was induced in 5 of 15 patients who had structural heart disease, and in none of the 18 patients who did not ( $p<0.05$ ). Polymorphic VT or ventricular fibrillation (VF) was induced in 5 of 15 patiehts $(33 \%)$ who had structural heart disease, and in 5 of 18 patients $(27 \%)$ who did not $(p>0.05)$. Patients who had inducible unimorphic VT were treated with antiarrhythmic drugs that suppressed the induction of VT, and 4 of 5 patients also received a pacemaker; no patient had a recurrence of syncope during followup. Patients who had inducible polymorphic VT and VF $(n=10)$ or no inducible VT $(n=18)$ received treatment directed at only carotid sinus syndrome. Two patients with inducible VT or VF and 1 patient without inducible VT had recurrent syncope during follow-up, but none had cardiac arrest or died suddenly. It is concluded that programmed stimulation should be performed in patients presumed to have carotid sinus syndrome who have structural heart disease; unimorphic VT may be induced in one-third of these patients, raising the possibility that $V T$ is the cause of syncope. Antiarrhythmic drug therapy may be unnecessary if polymorphic VT or VF is induced. In patients without heart disease, programmed stimulation is highly unlikely to yield a clinically significant arrhythmia.

(Am J Cardiol 1987;60:1073-1077)

A

patient who has had syncope may be presumed to have the carotid sinus syndrome if clinical evaluation demonstrates carotid hypersensitivity and no other apparent cause for syncope. However, clinical symptoms of syncope or near-syncope infrequently are reproduced by carotid sinus massage. ${ }^{1}$ Furthermore, carotid sinus hypersensitivity is associated with coronary artery disease ${ }^{2}$ and an abnormal electrocardiogram, ${ }^{3}$ and more than one-half of patients who have carotid hypersensitivity never have had syncope., ${ }^{2,4}$ Therefore, carotid sinus hypersensitivity may be an incidental finding unrelated to cerebral symptoms in patients who have had syncope or near-syncope, particular-

From the Division of Cardiology, Department of Internal Medicine, University of Michigan Medical Center, Ann Arbor, Michigan. Manuscript received March 10, 1987; revised manuscript received and accepted July 14, 1987.

Address for reprints: Fred Morady, MD, Division of Cardiology, University Hospital, 1500 East Medical center Drive, UH B1 F245-0022, Ann Arbor, Michigan 48109-0022.

ly in those who have structural heart disease. Programmed ventricular stimulation has been found to be useful in uncovering ventricular tachycardia (VT) as a potential cause of syncope in patients with unexplained syncope..$^{5-13}$ However, prior studies of programmed ventricular stimulation in patients with un. explained syncope have either excluded patients with carotid sinus hypersensitivity or included only a small number of such patients. ${ }^{10-12}$ Therefore, little data are available on the value of programmed ventricular stimulation in patients with syncope who have carotid sinus hypersensitivity. This study determines the results of programmed ventricular stimulation in patients with syncope or near-syncope and a presumptive diagnosis of carotid sinus syndrome. We wished to define the role of programmed ventricular stimulation in patients with presumed carotid sinus syndrome.

\section{Methods}

Characteristics of patients studied: The study group consisted of 28 men and 5 women with a history 
of syncope ( 28 patients) or near-syncope (5 patients) whose mean age ( \pm 1 standard deviation) was $66 \pm 10$ years The mean number of episodes of syncope or near-syncope was $2.3 \pm 1.4$. In each patient; a presumptive diagnosis of carotid sinus syndrome was based on the presence of carotid sinus hypersensitivity during carotid sinus massage, and the absence of any other apparent cause of syncope after a complete neurologic and noninvasive cardiac evaluation. Fourteen patients had coronary artery disease, 1 patient had an idiopathic dilated cardiomyopathy, and 18 patients did not have clinical evidence of structural heart disease.

All patients underwent at least 24 hours of continuous ambulatory electrocardiographic monitoring before undergoing an electrophysiologic study. Premature ventricular complexes were present in 24 patients, and 6 patients were found to have nonsustained VT (maximal duration 5 beats), but no patient had cerebral symptoms during monitoring.

Left ventricular ejection fraction was measured in 27 patients by either a radionuclide or contrast ventriculogram, or 2-dimensional echocardiogram. Left ventricular ejection fraction was normal (more than 0.50) in 17 patients and abnormal in 10 patients who had a mean ejection fraction of $0.33 \pm 0.11$.

Electrophysiologic study protocol: After informed consent was obtained, patients were studied in the fasting, unsedated state. Antiarrhythmic drug therapy was discontinued at least 4 half-lives before the electrophysiologic study. Quadripolar electrode catheters were inserted percutaneously in a femoral vein and positioned in the high right atrium, across the tricuspid valve to record the His bundle electrogram, against the right ventricular apex, and at either the right ventricular outflow tract or septum. A short cannula was inserted into a femoral artery for continuous monitoring of the arterial pressure. Electrocardiographic leads $V_{1}$, I and III, the intracardiac electrograms, and the arterial pressure tracing were recorded at a paper speed of 25 to $100 \mathrm{~mm} / \mathrm{s}$ on an Electronics for Medicine VR-12 or Siemens-Elema Minograf 7 recorder. Programmed stimulation was performed with a programmable stimulator (Bloom Associates, Litd.). The stimuli were $2 \mathrm{~ms}$ in duration and twice diastolic threshold.

Method of carotid sinus massage: Carotid sinus massage was performed with the patient supine during the course of electrophysiologic study: Carotid bruits were ruled out before carotid sinus massage. The cxaminer identified the fusiform carotid sinus or the area of greatest arterial pulsation lying anterior to the sternomastoid muscle at the upper border of the thyroid cartilage. Vigorous massage was performed for 5 seconds during continuous electrocardiographic and hemodynamic monitoring. After the heart rate and arterial pressure had returned to baseline values, the contralateral carotid sinus was massaged. If carotid sinus hypersensitivity was present, massage was repeated to assure reproducibility.

Patients were considered to have carotid sinus hypersensitivity if 1 of the 3 following responses occurred during carotid sinus massage ${ }^{1}$ : (1) cardioinhibitory re- sponse; more than 3 seconds of asystole, with minimal decrease in arterial pressure when carotid massage was repeated during ventricular pacing at a rate of 70 beats/min; (2) vasodepressor response; a decrease of $50 \mathrm{~mm} \mathrm{Hg}$ or more in the systolic arterial pressure, without a significant chänge in heart rate; or (3) a mixed response.

Atrial stimulation: The sinus node recovery time, atrioventricular conduction and the inducibility of supraventricular tachycardia were assessed by incremental atrial pacing and the atrial extrastimulus technique. In no patient was a potential cause of syncope uncovered by atrial pacing.

Programmed ventricular stimulation protocol: The programmed ventricular stimulation protocol has been described in detail previously. ${ }^{14}$ In brief, at least 2 basic drive cycle lengths were used $(600,500$ or 400 $\mathrm{ms}$ ). The basic drive trains were 8 beats in duration. Programmed stimulation with single and double extrastimuli was performed at the right ventricular apex, then at the right ventricular outflow tract or septum. If necessary, programmed stimulation with triple extrastimuli was then performed at the 2 right ventricular sites. Nonsustained VT was defined as VT 6 beats to 30 seconds in duration. Sustained VT was defined as VT lasting more than 30 seconds or requiring direct-current countershock. Electropharmacologic testing was performed in patients who had inducible sustained unimorphic VT.

Follow-up evaluation: All patients were seen periodically as outpatients or were contacted by telephone and were questioned about recurrences of syncope or near-syncope.

Statistical analysis: Statistical analyses were performed with Student $t$ test or Fisher exact test. A p value $<0.05$ was considered statistically significant.

\section{Results}

Response to carotid sinus massage: Seventeen patients had a cardioinhibitory response to carotid sinus massage, with a mean asystolic interval of $5.6 \pm 1.3$ seconds. Four patients had a vasodepressor response to carotid sinus massage, with a mean decrease in systolic arterial pressure at $76 \pm 13 \mathrm{~mm} \mathrm{Hg}$. Twelve patients had a mixed response.

Results of programmed ventricular stimulation: Unimorphic sustained VT (mean cycle length $274 \pm 49$ $\mathrm{ms}$ ) was induced by programmed stimulation with double extrastimuli in 4 patients and with triple extrastimuli in 1 patient (Table I). Sustained VF was induced in 1 patient by triple extrastimuli. Sustained polymorphic VT or ventricular flutter with a mean cycle length of $197 \pm 15 \mathrm{~ms}$ was induced in 2 patients by programmed stimulation with triple extrastimuli and in 1 patient with double extrastimuli. Polymorphic nonsustained VT (cycle length $224 \pm 28 \mathrm{~ms}$ and mean duration $6.3 \pm 4.8$ seconds) was induced in 6 patients by programmed stimulation with triple extrastimuli (Table II).

Comparison of patients with and without structural heart disease: Sustained unimorphic VT was in- 
TABLE I Clinical Characteristics and Follow-Up in Patients with Inducible Unimorphic Sustained Ventricular Tachycardia

\begin{tabular}{|c|c|c|c|c|c|c|c|}
\hline Patient & $\begin{array}{l}\text { Age (yr) } \\
\& \text { Sex }\end{array}$ & Heart Disease & LVEF & VT Morphology & $\mathrm{CL}(\mathrm{ms})$ & AA Therapy & $\begin{array}{c}\text { Duration of } \\
\text { Follow-Up (mo) }\end{array}$ \\
\hline 1 & $68 / \mathrm{M}$ & CAD & 0.20 & RBBB superior & 360 & $A+P P$ & 1 \\
\hline 2 & $73 / \mathrm{M}$ & GAD & 0.36 & RBBB superior & 260 & $A+P P$ & 7 \\
\hline 3 & $62 / M$ & CAD & 0.54 & LBBB superior & 260 & $A+P P$ & 12 \\
\hline 4 & $66 / M$ & $\mathrm{CAD}$ & 0.48 & LBBB superior & 250 & Quinidine & 16 \\
\hline 5 & $71 / \mathrm{M}$ & $D C$ & 0.40 & I inferior & 240 & Proc + PP & 5 \\
\hline
\end{tabular}

$\mathrm{A}=$ amiodarone; $\mathrm{AA}=$ antiarrhythmic $\mathrm{CAD}=$ coronary artery disease; $\mathrm{CHF}=$ congestive heart failure; $\mathrm{CL}=\mathrm{cycle}$ length; $\mathrm{DC}=$ dilated cardiomypathy; $\mathrm{I}$ $=$ indeterminant; $\mathrm{LBBB}=$ left bundle branch block; $\mathrm{LVEF}=$ left ventricular ejection fraction; $\mathrm{Proc}=$ procainamide; $\mathrm{PP}=$ permanent pacemaker; $\mathrm{RBBB}=$ right bundle branch block; VT = ventricular tachycardia.

TABLE II Clinical Characteristics and Follow-Up in Patients with Inducible Polymorphic Ventricular Tachycardia or Ventricular Fibrillation

\begin{tabular}{|c|c|c|c|c|c|c|c|}
\hline Patient & $\begin{array}{l}\text { Age (yr) } \\
\& \text { Sex }\end{array}$ & Heart Disease & LVEF & Type of VT & $\mathrm{CL}(\mathrm{ms})$ & AA Therapy & $\begin{array}{l}\text { Duration of } \\
\text { Follow-Up (mo) }\end{array}$ \\
\hline 6 & $80 / \mathrm{M}$ & $\mathrm{CAD}$ & 0.29 & Poly, NS & 250 & PP & 2 \\
\hline 7 & $62 / M$ & CAD & ND. & Poly, NS & 270 & $\mathrm{PP}$ & 9 \\
\hline 8 & $50 / M$ & CAD & 0.40 & Poly, NS & 195 & PP & 17 \\
\hline 9 & $54 / \mathrm{M}$ & CAD & 0.19 & $V$ flutter & 210 & 0 & 18 \\
\hline 10 & $64 / \mathrm{M}$ & CAD & 0.70 & VF & - & $\mathrm{PP}$ & 24 \\
\hline 12 & $69 / \mathrm{M}$ & 0 & ND & Poly, NS & 200 & 0 & 9 \\
\hline 13 & $79 / M$ & 0 & 0.89 & Poly, S & 200 & $P P$ & 20 \\
\hline 14 & $54 / F$ & 0 & $>0.50^{*}$ & Poly, NS & 200 & $\mathrm{PP}$ & 22 \\
\hline 15 & $75 / F$ & 0 & 0.61 & Poly, NS & 230 & 0 & 26 \\
\hline
\end{tabular}

*Echocardiographic determination.

$\mathrm{ND}=$ not done; $\mathrm{NS}=$ nonsustained; Poly $=$ polymorphic; $\mathrm{S}=$ sustained; VF = ventricular fibrillation: Other abbreviations as in Table $\mathrm{I}$.

duced in 5 of 15 patients who had structural heart disease, and in none of 18 patients who did not have structural heart disease $(\mathrm{p}<0.05)$. Polymorphic VT or VF was induced in 5 of 15 patients (33\%) who had structural heart disease and in 5 of 18 patients (27\%) who did not $(\mathrm{p}>0.05)$.

Comparison of patients with and without inducible unimorphic ventricular tachycardia: In the group of 15 patients who had structural heart disease, the 5 patients in whom sustained unimorphic VT was inducible were compared with 10 patients in whom unimorphic VT was not inducible. There were no significant differences between the 2 groups of patients in age or left ventricular ejection fraction. The patients in whom unimorphic VT was induced did not have a higher incidence of $\mathrm{Q}$ waves or bundle branch block on the electrocardiogram.

Treatment: A permanent paccmaker was implanted in 18 patients who had a cardioinhibitory response to carotid sinus massage and a history of either frequent episodes of syncope, syncope while driving or injury caused by syncope. A permanent pacemaker was not implanted in 15 patients because of either the presence of a significant vasodepressor component during carotid sinus massage or a history of only nearsyncope and not syncope.

Among the 5 patients who had inducible unimorphic sustained VT, 1 patient was treated with quinidine, 1 with procainamide and 3 with amiodarone. In each patient, the antiarrhythmic drug therapy wäs ef:fective in suppressing the induction of VT. Four of these 5 patients also underwent implantation of a permanent pacemaker. No patient in whom polymorphic VT or VF was induced was treated with antiarrhythmic drugs.

Follow-up: Among the 5 patients who had inducible unimorphic VT, 1 patient died from congestive heart failure after 1 month of follow-up and 4 patients have remained alive over a mean follow-up duration of $10 \pm 4$ months (range 5 to 16). No patient had syncope, near-syncope, cardiac arrest or sudden death during follow-up (Table I).

Among the 10 patients who had inducible polymorphic VT or VF, the mean duration of follow-up was 15 \pm 7 months (range 2 to 26 ) (Table II). One patient died from pneumonia. Two patients had a recurrence of syncope. In 1 of these patients, who had a vasodepressor response to carotid sinus massage, the features of the syncopal episode were characteristic of vasodepressor syncope. The second patient who had a recurrence of syncope had undergone implantation of a dual chamber pacemaker, and in this patient, the cause of the recurrent syncope was unclear. No patient in this group had a cardiac arrest or died suddenly during the follow-up period.

Eighteen patients without inducible arrhythmias were followed for a mean duration of $13 \pm 8$ months (range 2 to 31) (Table III). One patient died from non- 
TABLE III Clinical Characteristics and Follow-Up in Patients Without Inducible Ventricular Tachycardia

\begin{tabular}{|c|c|c|c|c|c|}
\hline Patient & $\begin{array}{l}\text { Age (yr) } \\
\& \text { Sex }\end{array}$ & Heart Disease & LVEF & AA Therapy & $\begin{array}{c}\text { Duration of } \\
\text { Follow-Up (mo) }\end{array}$ \\
\hline 16 & $59 / M$ & CAD & ND & PP & 2 \\
\hline 17 & $68 / M$ & CAD & 0.20 & 0 & 7 \\
\hline 18 & $46 / M$ & CAD & 0.72 & 0 & 16 \\
\hline 19 & $79 / M$ & CAD & 0.31 & PP & 19 \\
\hline 20 & $72 / M$ & CAD & 0.75 & PP & 31 \\
\hline 21 & $72 / F$ & 0 & ND & PP & 3 \\
\hline 22 & $75 / F$ & 0 & $>0.50^{*}$ & 0 & 5 \\
\hline 23 & $71 / \mathrm{M}$ & 0 & 0.47 & 0 & 8 \\
\hline 24 & $53 / M$ & 0 & 0.52 & PP & 9 \\
\hline 25 & $63 / M$ & 0 & ND & 0 & 10 \\
\hline 26 & $72 / \mathrm{M}$ & 0 & $>0.50^{*}$ & $\mathrm{PP}$ & 13 \\
\hline 27 & $61 / M$ & 0 & $>0.50^{*}$ & PP & 13 \\
\hline 28 & $58 / \mathrm{M}$ & 0 & $>0.50^{*}$ & PP & 14 \\
\hline 29 & $44 / M$ & 0 & 0.79 & 0 & 13 \\
\hline 30 & $51 / \mathrm{M}$ & 0 & $>0.50^{*}$ & 0 & 15 \\
\hline 31 & 72/M & 0 & ND & 0 & 16 \\
\hline 32 & $72 / \mathrm{M}$ & 0 & $>0.50^{*}$ & 0 & 23 \\
\hline 33 & $86 / F$ & 0 & 0.73 & 0 & 31 \\
\hline
\end{tabular}

*Echocardiographic determination.

Abbreviations as in Tables I and II.

cardiac causes. One patient had recurrent syncope. No patient in this group had a cardiac arrest or died suddenly during the follow-up period.

\section{Discussion}

Role of programmed ventricular stimulation in patients presumed to have carotid sinus syndrome: The results of this study suggest that programmed ventricular stimulation may play an important role in the management of some patients with syncope or nearsyncope who are presumed to have the carotid sinus syndrome based on the finding of carotid sinus hypersensitivity and the absence of any other likely cause of syncope after a neurologic and noninvasive cardiac evaluation. Among patients with structural heart disease but no clinical evidence of VT as the cause of syncope, programmed ventricular stimulation may result in the induction of sustained unimorphic VT in one-third of patients.

Inducible sustained unimorphic VT has been demonstrated to be a clinically important finding in prior studies of patients with unexplained syncope. .,11 $^{8}$ Accordingly, patients in this study who had inducible unimorphic VT were treated with antiarrhythmic drugs demonstrated during electropharmacologic testing to be effective in suppressing the induction of VT. This antiarrhythmic drug therapy may have contributed to prevention of recurrent syncope during the follow-up period. Because programmed ventricular stimulation may uncover a second potential cause for syncope in up to one-third of patients with syncope and carotid sinus hypersensitivity who have structural heart disease, programmed ventricular stimulation is appropriate in these patients, unless the diagnosis of carotid sinus syndrome has been established by electrocỏardiographic monitoring during a spontaneous episode of syncope.
None of the 18 patients in this study who did not have structural heart disease were found to have inducible sustained unimorphic VT. Similarly, in prior studies of electrophysiologic testing in patients with unexplained syncope, sustained unimorphic VT was a rare finding in patients without structural heart disease. ${ }^{5-12}$ Therefore, because programmed ventricular stimulation is likely to have an extremely low yield of clinically important VT in patients without underlying heart disease who are presumed to have the carotid sinus syndrome, programmed ventricular stimulation need not be performed in these patients unless there is a specific reason to suspect VT as the cause of syncope based on the history or results of electrocardiographic monitoring.

Patients with structural heart disease most likely to have inducible unimorphic ventricular tachycardia: Analysis of clinical variables demonstrated that among the 15 patients with structural heart disease, there were no significant differences between the patients who had inducible unimorphic VT and those who did not. Therefore, it may not be possible to predict on the basis of clinical evaluation which patients with structural heart disease and presumed carotid sinus syndrome are likely to have inducible unimorphic VT. Unless the diagnosis of carotid sinus syndrome has been established on clinical grounds instead of being a presumptive diagnosis, programmed ventricular stimulation should be considered in all patients who have structural heart disease.

Significance of polymorphic ventricular tachycardia and ventricular fibrillation: Prior studies in patients with and without structural heart disease who either were asymptomatic or had a history of unexplained syncope have demonstrated that inducible polymorphic VT and VF in these patients most likely represent a laboratory artifact devoid of clinical signif- 
icance. $8,11,15-20$ Therefore, the patients in this study who had inducible polymorphic VT or VF were not treated with antiarrhythmic drugs. Although 2 of these patients had a recurrence of syncope, the historical details in 1 patient were typical of vasodepressor syncope. None of these patients died suddenly or had symptomatic VT during follow-up. This suggests that inducible polymorphic VT or VF may not have any prognostic significance in this patient group and may not necessarily be an indication for antiarrhythmic drug therapy.

Results of previous studies: In 3 prior reports, a total of 23 patients who had syncope or near-syncope and carotid hypersensitivity underwent programmed ventricular stimulation with up to 2 extrastimuli. ${ }^{10-12}$ In contrast to the results of the present study, polymorphic VT was induced in only 2 of these 23 patients and unimorphic VT was induced in only 1 . The higher incidence of inducible polymorphic VT in the present study undoubtedly is related to the use of triple extrastimuli. However, 4 of the 5 unimorphic VTs in the present study were induced by 2 extrastimuli; therefore the higher incidence of inducible unimorphic VT cannot be attributed to our use of more extrastimuli. Prior reports did not always specify which patients with carotid hypersensitivity had structural heart disease. Therefore, it is possible that the higher incidence of inducible unimorphic VT in the present study is related to a higher incidence of structural heart disease in our patient group.

Limitations: The major limitation of this study is one that is inherent to all studies of patients with unexplained syncope. Because none of the patients in this study was undergoing electrocardiographic monitoring at the time of syncope, the diagnosis of unimorphic VT as the cause of syncope on the basis of electrophysiologic testing was presumptive and not based on definitive evidence. Although prior studies have demonstrated that inducible sustained unimorphic VT is an unusual finding in patients who have not had unimorphic VT, ${ }^{15-17}$ we cannot rule out the possibility that sustained unimorphic VT was an incidental finding unrelated to syncope in the patients in this study. Because of a possible risk of sudden death, this study did not include a control group of patients who had inducible sustained unimorphic VT and were not treated with antiarrhythmic drugs. Furthermore, because 4 of 5 patients who had inducible sustained unimorphic VT and were treated with antiarrhythmic drugs also underwent implantation of a permanent pacemaker, it cannot be determined whether the lack of recurrent syncope during follow-up was attributable to the antiarrhythmic drugs, the pacemaker, or both.

\section{References}

1. Thonas JE. Hyperuclive curotid sinus reflex and carotid sinus syncope. Mayo Clin Proc 1969:44:127-139.

2. Brown KA, Maloney JD, Smith HC, Hartzler GO, Ilstrup DM. Carotid sinus reflex in patients undergoing coronary angiography: relationship of dcgrco and location of coronary artery disease to response to carotid sinus massage. Circulation 1980;62:697-703.

3. Smuddy J. Lewis D. Dunn M. The effect of carotid sinus massage in older men. I Gerontol 1972;27:209-211.

4. Nathanson MH. Hyperactive cardioinhibitory carotid sinus reflex. Arch Intern Med 1946:77:491-503.

5. DiMarco JP, Garan H, Harthorne JW, Ruskin IN. Intracardiac electrophysiologic techniques in recurrent syncope of unknown cause. Ann Intern Med 1981;95:542-548.

6. Hess DS, Morady F, Scheinman MM. Electrophysiologic testing in the evaluation of patients with syncope of undetermined etiology. Am I Cardiol 1982;50:1309-1315.

7. $\Lambda$ khtar M, Shenasa M, Denker S, Gilbert CJ, Rizwi N. Role of cardiac electrophysiologic studies in patients with unexplained recurrent syncope. PACE 1983;6:192-201.

8. Morady F, Shen E, Schwartz A, Hess D, Bhandari A, Sung RJ, Scheinman MM. Long term follow-up of patients with recurrent unexplained syncope evaluated by electrophysiologic testing. JACC 1983;2:1053-1059.

9. Morady F, Higgins J, Peters RW, Schwartz AB, Shen EN, Bhandari A Scheinman MM, Sauve MJ. Electrophysiologic testing in bundle branch block and unexplained syncope. Am I Cardiol 1984:54:587-591.

10. Olshansky B, Mazuz M, Martins JB. Significance of inducible tochycardio in patients with syncope of unknown origin:a long-term follow-up. JACC 1985;5:216-223.

11. Doherty JU, Pembrook-Rogers D, Grogan EW, Falcone RA, Buston AE Marchlinski FE, Cassidy DM, Kienzle MG, Almendral JM, Josephson ME. Electrophysiologic evaluation and follow-up characteristics of patients with recurrent unexplained syncope and presyncope. Am / Cardiol 1985;55:703708 .

12. Denes P, Ezri M. The role of electrophysiologic studies in the management of patients with unexplained syncope. PACE 1985;8:424-435.

13. Teichman SL, Felder SD, Matos [A, Kim SG, Waspe I.E, Fisher ID. The value of electrophysiologic studies in syncope of undetermined origin: report of 150 cases. Am Heart I 1985;2:469-479.

14. de Buitleir M, Morady F, DiCarlo LA, Baerman JM, Krol RB. Immediate reproductibility of clinical and nonclinical forms of induced ventricular tachycardia. Am I Cardiol 1986:58:279-282.

15. Brugada P, Abdollah $\mathrm{H}$, Heddle B, Wellens HJJ. Results of a ventricular stimulation protocol using a maximum of 4 premature stimuli in patients without documented or suspected ventricular arrhythmias. Am I Cardiol 1983;52:1214-1218.

16. Morady F, Shapiro W, Shen E, Sung RJ, Scheinman MM. Programmed ventricular stimulation in patients without spontaneous ventricular tachycardia. Am Heart I 1984;107:875-882.

17. Brugada $\mathrm{P}$, Green M, Abdollah $\mathrm{H}$, Wellens HJ]. Significance of ventricular arrhythmias initiated by programmed ventricular stimulation the impor tance of the type of ventricular arrhythmia induced and the number of premature stimuli required. Circulation 1984:69:87-92.

18. DiCarlo LA Jr, Morady F, Schwartz AB, Shen EN, Baerman JM, Krol RB Scheinman $M M$, Sung RJ. Clinical significance of ventricular fibrillationflutter induced by ventricular programmed stimulation. Am Heart I 1985; 109:959-963.

19. Mahmud R, Denker S, Lehmann MH, Tchou P, Dongas J, Akhtar Mi Incidence and clinical significance of ventricular fibrillation induced with single and double ventricular extrastimuli. Am I Cardiol 1986;58:75-79.

20. Wellens HIJ, Brugada P, Stevenson WG. Programmed electrical stimula tion of the heart in patients with life-threatening ventricular arrhythmias: what is the significance of induced arrhythmias and what is the correct stimulation protocol? Girculation 1985:72:1-7. 